\title{
Brain death induces the alteration of liver protein expression profiles in rabbits
}

\author{
BING DU $^{1 *}$, LING LI $^{1 *}$, ZHIBIAO ZHONG $^{1}$, XIAOLI FAN ${ }^{1}$, BINGBING QIAO $^{1}$, \\ CHONGXIANG HE ${ }^{1}, \mathrm{ZHEN} \mathrm{FU}^{1,2}$, YANFENG WANG ${ }^{1}$ and QIFA YE $^{1,2}$
}

\author{
${ }^{1}$ Zhongnan Hospital of Wuhan University, Institute of Hepatobiliary Diseases of Wuhan University, \\ Transplant Center of Wuhan University, Hubei Key Laboratory of Medical Technology on Transplantation, \\ Wuhan, Hubei 430071; ${ }^{2}$ The Third Xiangya Hospital of Central South University, Research Center of National \\ Health Ministry on Transplantation Medicine Engineering and Technology, Changsha, Hunan 410013, P.R. China
}

Received February 18, 2014; Accepted May 30, 2014

DOI: $10.3892 /$ ijmm.2014.1806

\begin{abstract}
At present, there is no accurate method for evaluating the quality of liver transplant from a brain-dead donor.Proteomics are used to investigate the mechanisms involved in brain death-induced liver injury and to identify sensitive biomarkers. In the present study, age- and gender-matched rabbits were randomly divided into the brain death and sham groups. The sham served as the control. A brain-death model was established using an intracranial progressive pressurized method. The differentially expressed proteins extracted from the liver tissues of rabbits that were brain-dead for $6 \mathrm{~h}$ in the two groups were determined by two-dimensional gel electrophoresis and matrix-assisted laser desorption ionization time of flight mass spectrometry. Although there was no obvious functional and morphological difference in 2,4 and $6 \mathrm{~h}$ after brain death, results of the proteomics analysis revealed $973 \pm 34$ and $987 \pm 38$ protein spots in the control and brain death groups, respectively. Ten proteins exhibited a $\geq 2$-fold alteration. The downregulated proteins were: aldehyde dehydrogenase, runt-related transcription factor 1 (RUNX1), inorganic pyrophosphatase, glutamate-cysteine ligase regulatory subunit and microsomal cytochrome B5. By contrast, the expression of dihydropyrimidinase-related protein 4 , peroxiredoxin-6, 3-phosphoinositide-dependent protein kinase-1, 3-mercaptopyruvate and alcohol dehydrogenase were clearly upregulated. Immunohistochemistry and western blot analysis results revealed that the expression of RUNX1 was gradually
\end{abstract}

Correspondence to: Dr Qifa Ye, Zhongnan Hospital of Wuhan University, Institute of Hepatobiliary Diseases of Wuhan University, Transplant Center of Wuhan University, Hubei Key Laboratory of Medical Technology on Transplantation, 169 Donghu Road, Wuhan, Hubei 430071, P.R. China

E-mail: yqf_china@163.com

*Contributed equally

Key words: brain death, proteomics, runt-related transcription factor 1 , liver increased in a time-dependent manner in 2, 4, and $6 \mathrm{~h}$ after brain death. In conclusion, alteration of the liver protein expression profile induced by brain death indicated the occurrence of complex pathological changes even if no functional or morphological difference was identified. Thus, RUNX1 may be a sensitive predict factor for evaluating the quality of brain death donated liver.

\section{Introduction}

Brain death refers to the irreversible loss of all functions of the brain, including the brainstem. Legal recognition of donor sources, as well as the expansion of origin of the donor source are crucial factors that remain to be addressed $(1,2)$. Since the establishment of the first brain-death donor model in the late 1960s and early 1970s, solid organ transplantation from brain-dead donors has become common in western countries (3). In China, the rapid progress in organ transplant legislation and establishment of voluntary donation, donation after brain death (DBD) or cardiac death (DCD) has gradually replaced deceased donors and is due to become the main source of donors (4-6). However, the quality of brain-dead donors was comparatively worse than that of deceased donors. Subsequently, recent or long-term transplant efficacy was not successful (7-9).

Numerous studies have reported that the changes of hemodynamics and metabolic parameters $(10,11)$, hormonal changes and the endocrines (12-14), the consumption of coagulation factors $(15,16)$, the release of inflammatory cytokines and the change of immunological states $(10,17-20)$ may be responsible for the injury of brain death-donated organs. However, the detailed mechanism involved remains to be clarified. Additionally, accurate methods for identifying the quality of brain death donor organs remain to be identified.

Protein is considered the ultimate performer of various biological functions and proteomics is the classical method used for the screening of specific biological markers $(21,22)$. Using two-dimensional gel electrophoresis and matrix-assisted laser desorption ionization time of flight mass spectrometry, the purpose of this study was to detect alterations in the liver 
protein expression profile between the brain death and control groups at different time-points after brain death, and to predict sensitivity factors to explain the detailed mechanism involved. Additionally, we aimed to establish a sensitivity method to evaluate the quality of brain death donors for transplantation.

\section{Materials and methods}

Animals. Twelve-week-old male rabbits (Wuhan Wanqianjiahe Experimental Animal Breeding Center) were randomly divided in the brain death and sham (control) groups. Each group was subdivided into four subgroups according to different time-points $(2,4,6$ and 8 h) after brain death $(n=5)$. All the rabbits were kept in the room with water and food ad libitum in a room with controlled temperature $\left(22 \pm 1^{\circ} \mathrm{C}\right)$, humidity $(50-70 \%)$ and 12 -h light/dark cycle in the Experimental Animal Center of Wuhan University. Animal experiments were conducted under Institutional guidelines and approved by the Ethics Committee for Animal Care and Use of the Wuhan University according to the animal protocol.

Establishment of the model of rabbit brain death. A brain death model was established using an intracranial progressive pressurized method, similar to that of Pratschke et al (23). After being anesthetized with pentobarbital sodium at a dose of $40 \mathrm{mg} / \mathrm{kg}$, the rabbits were placed on the operating table in a supine position. Femoral artery and vein cannulation, xiphoid separation and tracheal intubation were performed, as well as burr hole and catheter placement. Vital signs of the rabbits including electrocardiogram, blood pressure, respiratory and electroencephalogram were monitored using a biological functional system, rodent ventilator and intelligent temperature control instrument (Thai Union Technology, Co., Ltd., Chengdu, China). Intracranial pressure was increased as required until the occurrence of brain death.

Liver function measuring. Blood samples were collected from each rabbit at 2, 4, 6 and $8 \mathrm{~h}$ after brain death. Serum glutamic pyruvic transaminase (ALT) and glutamic oxaloacetic transaminase (AST) levels reflecting the liver functions were measured by automatic biochemical analyzer (Hitachi, Tokyo, Japan).

Histomorphometrical evaluation. At 2, 4, 6 and $8 \mathrm{~h}$ after brain death, the liver tissues were isolated and fixed in $10 \%$ buffered formaldehyde for $>24 \mathrm{~h}$ and then embedded in paraffin. Serial sections $(4 \mu \mathrm{m})$ were stained with hematoxylin and eosin for cell morphometry. Three sections per animal and five fields per section were scanned and computerized with a digital image analyzer [Medical Image Analysis System (MIAS)] (Beijing University of Aeronautics and Astronautics).

Protein extraction and 2-DE proteomics profiling. Following the manufacturer's instructions, the ReadyPrep Sequential Extraction kit (Bio-Rad, Hercules, CA, USA) was used to extract proteins from the liver tissues. The tissues were then washed with the PlusOne 2-D Clean-Up kit (GE Healthcare, Piscataway, NJ, USA) and dissolved with sample buffer. Proteins $(150 \mu \mathrm{g})$ from the control group and the 6-h after brain death group were mixed with rehydration buffer. Using an Ettan IPGphor Electrophoresis System (GE Healthcare), the mixture was isoelectrically focused at $500 \mathrm{~V}$ for $1 \mathrm{~h} ; 1,000 \mathrm{~V}$ for $1 \mathrm{~h} ; 3,000 \mathrm{~V}$ for $1 \mathrm{~h}$ and $8,000 \mathrm{~V}$ for $9.5 \mathrm{~h}$ subsequent to rehydration for $12 \mathrm{~h}$ at $30 \mathrm{~V}$ on Immobiline IPG DryStrips (GE Healthcare). IPG strips were applied for $12 \%$ sodium dodecyl sulfate polyacrylamide gel electrophoresis (SDS-PAGE) using a PROTEAN ${ }^{\circledR}$ II xi Cell system (Bio-Rad) following equilibration for $2 \times 15$ min in an equilibration buffer. Each sample was measured in triplicate.

Gel image acquisition and analysis. The Coomassie Brilliant Blue R-350 (Amersham Biosciences, Amersham, UK) and PDQuest 2D analysis software (Bio-Rad) were used to stain 2-DE gel images and detect protein spots, respectively. Sensitivity parameters were simultaneously reproduced for each gel image and spot detection and matching were manually revised in the software. Based on the three independent pools of biological material, reproducible protein patterns were measured by the presence of each individual protein in three replicate gels. The intensity of each protein spot was normalized to the total density in the gel.

Protein identification. Following excision from the gel, the protein spots were subjected to destaining, washing and in-gel digestion with protease trypsin at $37^{\circ} \mathrm{C}$ overnight. Subsequently, peptides were extracted from the gel and dried by centrifugal lyophilization. The peptide mixtures were redissolved in $0.5 \%$ TFA and analyzed using a 4700 Proteomics Analyzer (Applied Biosystems, Inc., Foster City, CA, USA). The Mascot software (Matrix Science, London, UK) was used for protein identification and the mass spectra were searched in the Swiss-Prot protein database. Protein scores $>56$ were considered as significant. If one spot matched $>1$ protein member, the one with the highest score was taken into consideration.

Re-identification of typical protein. In order to further identify these different proteins, randomly runt-related transcription factor 1 (RUNX1) was selected and the difference with immunohistochemistry and western blot analysis in the brain death and control groups was re-identified.

The liver tissues obtained from the control and experimental groups at 2, 4, 6 and $8 \mathrm{~h}$ after brain death were deparaffinized and the endogenous peroxidase was blocked with $3 \% \mathrm{H}_{2} \mathrm{O}_{2}$ for $10 \mathrm{~min}$. The tissues were then incubated with primary polyclonal rabbit antibody against RUNX1 (1:500) and $\beta$-actin (1:200; Boster Biological Engineering, Co., Ltd., Wuhan, China) for $1 \mathrm{~h}$ at $37^{\circ} \mathrm{C}$, followed by biotin-labeled goat anti-rabbit immunoglobulin ( $\mathrm{Ig}$ ) $\mathrm{G}$ for $20 \mathrm{~min}$. After sequential incubation with Streptavidin-Biotin complex (SABC) (Santa Cruz Biotechnology, Inc., Santa Cruz, CA, USA) and DAB as substrate, the samples were counterstained with hematoxylin. Five random fields of each stained section were visualized and analyzed using morphometric software (MIAS, Beijing University of Aeronautics and Astronautics) by an investigator who was blinded to the animals' treatment status.

The proteins extracted from the liver tissues of the control and brain death groups at 2, 4, 6 and $8 \mathrm{~h}$ were prepared by homogenizing in RIPA buffer containing protease inhibitors (Boston BioProducts, Inc., MA, USA) followed by centrifugation at $10,000 \mathrm{x}$ g for $10 \mathrm{~min}$ at $4^{\circ} \mathrm{C}$. Samples were 

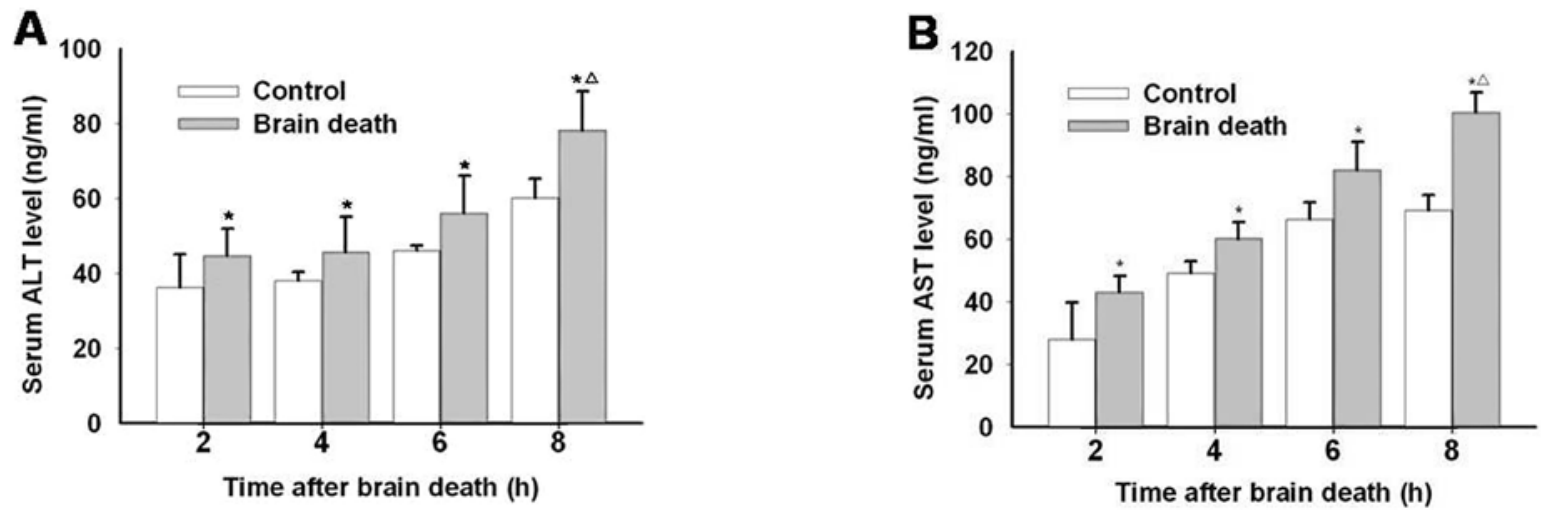

Figure 1. Effects of brain death at different time-points on alteration of serum glutamic pyruvic transaminase (ALT) and glutamic oxaloacetic transaminase (AST) levels ( $\mathrm{n}=5$ ). The serum ALT and AST levels in the control and brain death groups at 2, 4,6 and $8 \mathrm{~h}$ after brain death were measured by automatic biochemical analyzer, separately. (A) and (B) Although a gradual increase for the brain death groups in a time-dependent manner is evident, the serum ALT and AST levels show no significant differences at 2, 4, and $6 \mathrm{~h}$ after brain death as compared to the control groups. However, a significant difference is evident between the brain death and control groups at $8 \mathrm{~h} .{ }^{*} \mathrm{P}<0.05$ indicates statistical significance compared with previous groups, ${ }^{\wedge} \mathrm{P}<0.05$ indicates statistical significance compared with control groups.

stored at $-80^{\circ} \mathrm{C}$ until use. Protein concentrations of the lysates were determined by the BCA Assay kit (Pierce Biotechnology, Inc., Rockford, IL, USA). Proteins were then separated using $12.5 \%$ SDS polyacrylamide gel electrophoresis and transferred to a PVDF membrane ( $0.2 \mu \mathrm{m}$; Millipore, Bedford, MA, USA). The membrane was then blocked with $5 \%$ non-fat dry milk overnight and probed with primary rabbit polyclonal antibody against RUNX1 (1:500, Boster Biological Engineering, Co., Ltd.). The proteins were then detected on the blot using infrared-labeled secondary antibodies visualized in $800 \mathrm{~nm}$ fluorescence channels. The blot was developed and quantified using the Odyssey Infrared Imaging System (LI-COR Biosciences, Lincoln, NE, USA) following the manufacturer's instructions.

Statistical analysis. Data were expressed as the mean \pm standard deviation (SD). Calculations were performed using SPSS 18.0 software. One-way ANOVA was used to compare the differences between two groups. The intergroup differences were compared with repeated measurement design. $\mathrm{P}<0.05$ was considered statistically significant.

\section{Results}

Alteration of liver function. Compared with the previous group, there were obvious differences for serum ALT and AST level in each brain death group from initiation to $8 \mathrm{~h}$ after rabbits' brain death. Compared with the control groups, no marked changes in the ALT and AST levels were observed for the 2, 4 and $6 \mathrm{~h}$ brain death groups. However, a significant difference between the two groups was observed at $8 \mathrm{~h}$ (Fig. 1).

The morphological alteration of liver. No obvious morphological alteration occurred for the liver cells for the control groups at 2, 4 and $6 \mathrm{~h}$. However, some inflammatory cell infiltration occurred for the 8-h groups. For the brain death groups, the liver cells were almost normal in the 2 -h group, while mild edema, osteoporosis and compression of the hepatic sinus part was evident in the 4-h group. Liver injuries gradually became exacerbated in a time-dependent manner. In particular, the ballooning degeneration, sinusoidal pressure, no significant hepatic cord structure, abundant periportal lymphocytic infiltration and part of focal necrosis were found in all 8-h brain death group livers (Fig. 2).

2-DE proteomics profiling of different proteins. Different proteins were obtained by analyzing and comparing the 2-DE-based proteomic profiling of the control and brain death group at $6 \mathrm{~h}$ after brain death. PDQuest 2D analysis software was employed to detect $973 \pm 34$ protein spots in the control group and $987 \pm 38$ protein spots in the brain death group. Results of the statistical analysis of 2-DE-based proteomic profiling revealed that there were 52 obvious different protein spots between the control and brain death group. Ten of the 52 protein spots differentially expressed in a $>2$-fold increase or decrease were identified by MS/MS analysis. The positions on the 2-DE-based profiling were annotated in Fig. 3.

Mass spectrum identification and the function classification of different proteins. Following MALDI-TOF/TOF tandem mass spectrometry analysis and the Swiss-Prot protein database search, we found that 5/10 identified different proteins were upregulated while the remaining five were downregulated. The biological associations between the alterations of identified proteins and the progression of brain death-induced liver injury were searched on the www.uniprot.org according to the individual biological and molecular functions. The major biological functions of these 10 proteins were divided into six classifications, including material metabolism $(3 / 10)$ and redox regulation $(2 / 10)$, energy metabolism $(1 / 10)$, cell proliferation and differentiation (3/10), lipid metabolism (1/10) and detoxification (2/10) and neurodevelopment (1/10). Basic information of these proteins and their classifications are listed in detail in Table I.

Identification and re-identifications of RUNX1 proteins. The predicted molecular mass/isoelectric point $(\mathrm{pI})$ value for RUNX1 was $49 \mathrm{kDa} / 9.08$ which was suitable to the position 
a

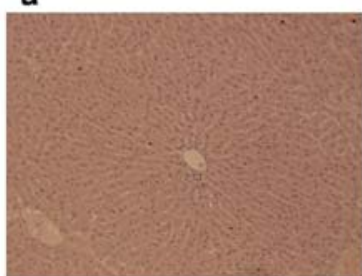

e

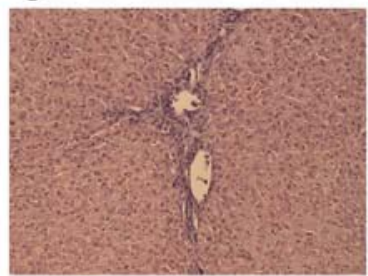

b

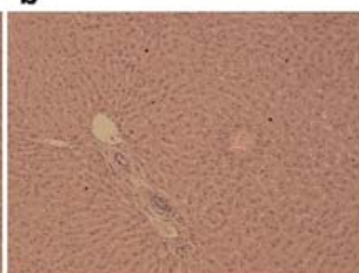

f

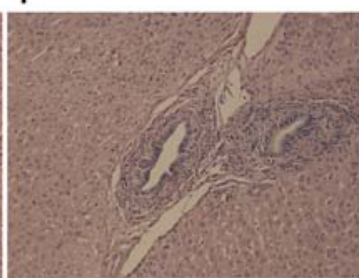

c

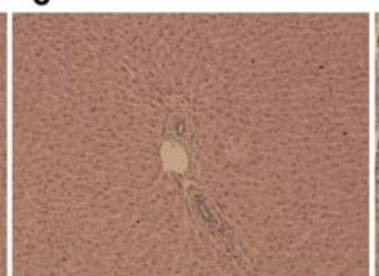

g

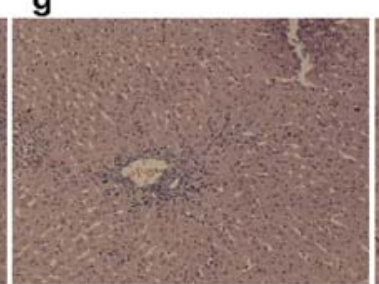

d

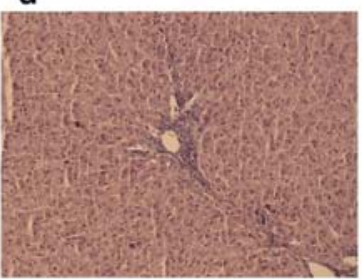

h

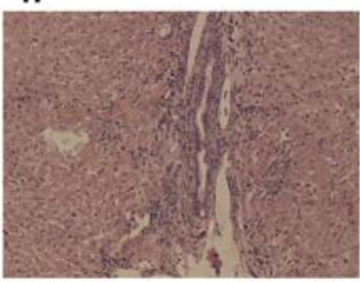

Figure 2. Effects of brain death at different time-points on the morphological alteration of liver ( $\mathrm{n}=5$ ). Alterations of morphometry of liver cells stained by hematoxylin and eosin (x100) are shown in Fig. 2. (a-d) No obvious differences were evident in (a-c) at 2, 4, 6 and $8 \mathrm{~h}$ for the control group, whereas some inflammatory cell infiltration is evident in (d). (e-h) Morphological alterations in liver at 2, 4, 6 and $8 \mathrm{~h}$ for the brain death group. Liver cells were almost normal in the (e) brain death group, while mild edema, osteoporosis and compression of the hepatic sinus part were observed in the liver of the (f) group. Obvious ballooning degeneration, sinusoidal pressure, no significant hepatic cord structure, abundant periportal lymphocytic infiltration and part of focal necrosis were found in $(\mathrm{g})$ and $(\mathrm{h})$ of the livers of the brain death group.

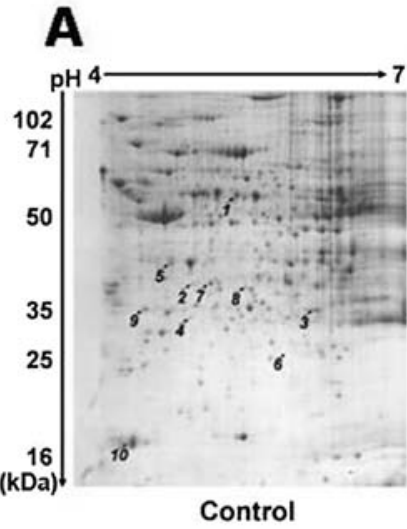

Control

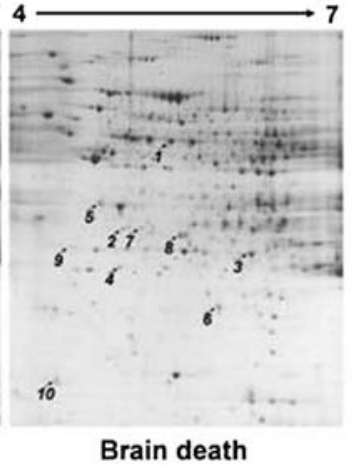

Brain death
B

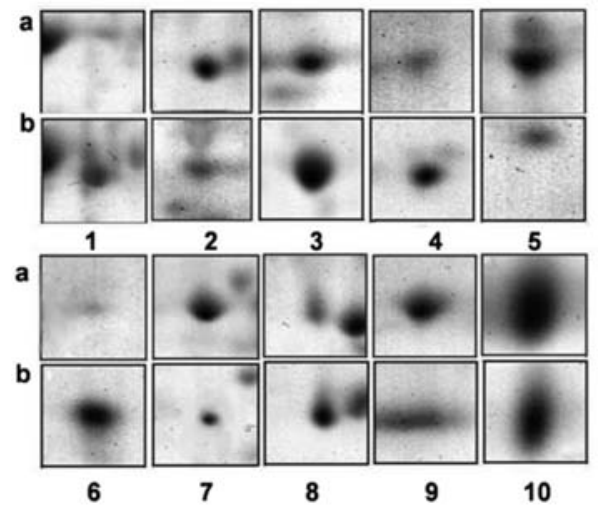

Figure 3. Differential protein expression profile analysis of the control and brain death groups using 2-DE gels (n=5). Total protein lysates were separated by isoelectric focusing (IEF) using IPG strips $(18 \mathrm{~cm}, \mathrm{pH} 4-7)$ in the first dimension followed by $12 \%$ sodium dodecyl sulfate polyacrylamide gel electrophoresis (SDS-PAGE) in the second dimension. Coomassie-stained gels were analyzed using PDQuest 2D analysis software. (A) Ten of 52 differentially expressed spots were identified by MS/MS analysis (marked with arrows and numbers). (B) Local enlarged 2-DE gel images of 10 spots in brain death liver tissues (b1-10) and controls (a1-10).

of the corresponding spot (spot 5) on the 2-DE gel. MS/MS analysis showed that RUNX1 was identified with a Mascot score of 67 and $29 \%$ sequence coverage (Fig. 4).

Results of the immunohistochemical analysis of RUNX1 revealed that the expression of RUNX1 was decreased in a time-dependent manner in the brain death group. Additionally, the level of RUNX1 for each time-point to some extent decreased compared with the control groups (Fig. 5A). Similarly, results of the western blot analysis revealed a gradual decrease in the brain death group at each time-point compared with control group (Fig. 5B).

\section{Discussion}

Previously, the detailed mechanism involving the effect of brain death on the quality of the transplant donor was unclear. In their study, Van Der Hoeven et al (24) used a rat brain death model and found that brain death-induced injury is associated with apoptosis. Avlonitis et al (10) also demonstrated that brain death in rats induced an inflammatory response represented by the elevated levels of IL-6, TNF- $\alpha$, neutrophil CD11b/CD18, cytokine-induced neutrophil chemoattractant (CINC)-1 and CINC-3. Similar to the establishment of the pig brain death model (25), in the present study, a new rabbit brain death model using an intracranial progressive pressurized method was established using the biological functional system, rodent ventilator and intelligent temperature control instrument. Maintaining of continuous breathing and the monitoring of electroencephalography ensured the model was similar to the state of brain death utilized in the clinic.

Weiss et al (26) reported that significantly upregulated levels of MIP-1 $\alpha$, IL-4, IFN- $\gamma, \mathrm{HO}-1$, CD3 and CD25 in brain death donor transplantation may be due to the phase of ischemic 


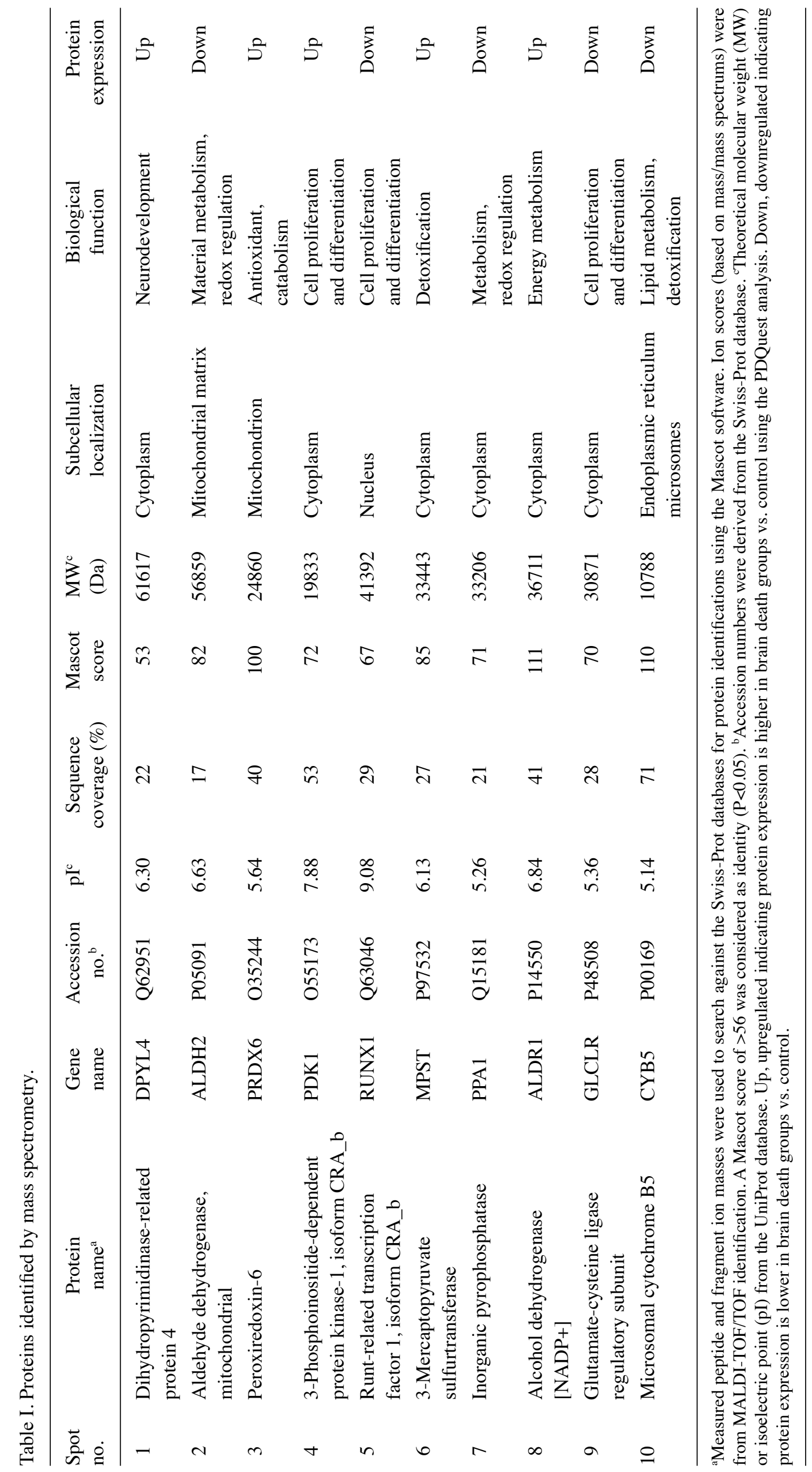




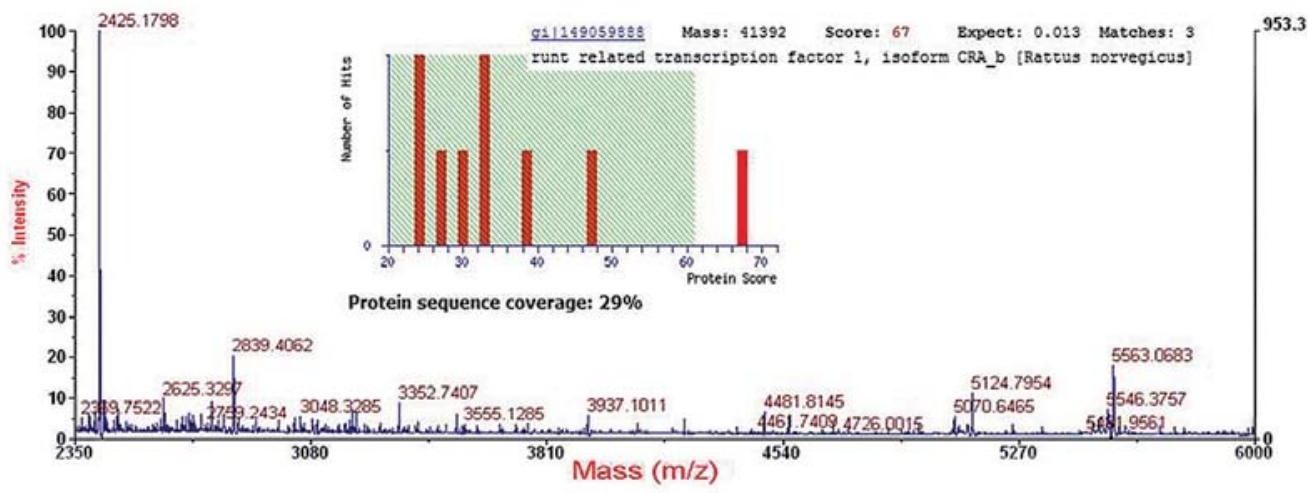

Figure 4. Identification of alteration of runt-related transcription factor 1 (RUNX1) protein expression by MALDI-TOF/TOF tandem mass spectrometry. Acquired spectra were searched against a Mascot search engine based on the Swiss-Prot protein database. MS/MS spectrum of the precursor ion with $\mathrm{m} / \mathrm{z} 2425.2$ for peptide 335ILPPCTNASTGAALLNPSLPSQSDVVETEGSHSNSPTNMPPARLEEAVWRPY386 with corresponding peak values was identified as RUNX1. Inset is the Swiss-Prot protein score from the Swiss-Prot database.
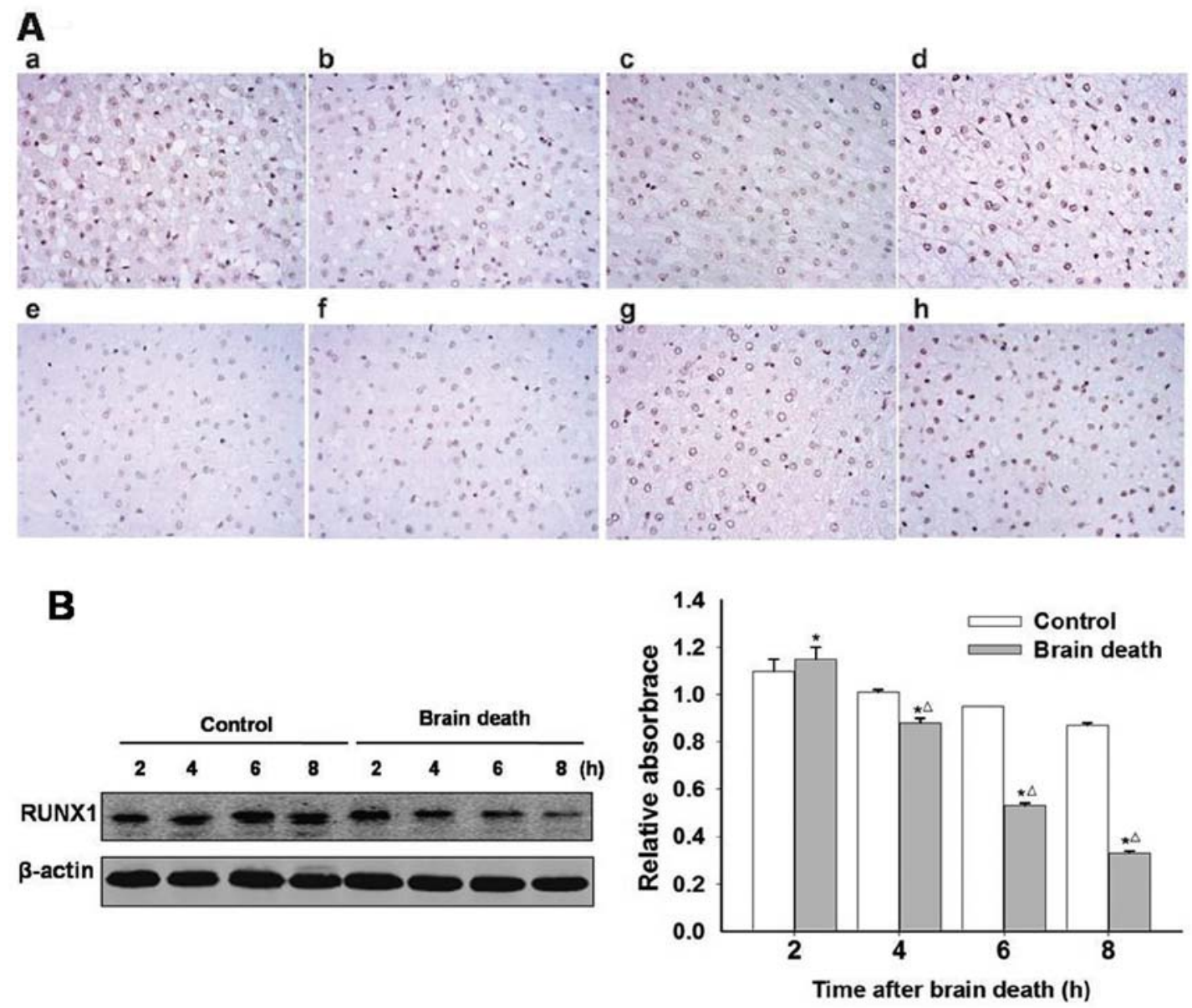

Figure 5. Re-identification of altenation of runt-related transcription factor 1 (RUNX1) proteins by immunohistochemistry and western blot analysis. (A) Representative images (x100) of immunohistochemical analysis of (a-d) control samples and (e-h) paired brain death groups. The protein expression of RUNX1 stained in the nucleus in each brain death group is clearly decreased as compared to the control group at 2, 4, 6 and $8 \mathrm{~h}$ after brain death (B) Representative results of western blot analysis of paired brain death groups and control samples with $\beta$-actin as an internal control. A significant decrease in a time-dependent manner and compared with the control groups was observed for the brain death groups. ${ }^{*} \mathrm{P}<0.05$ indicates statistical significance compared with the previous groups. ${ }^{\triangle} \mathrm{P}<0.05$ indicates statistical significance compared with the control groups.

reperfusion prior to transplantation. In the present study, we have demonstrated that from initiation to $6 \mathrm{~h}$ after brain death, there were no obvious functional or morphological alterations (Figs. 1 and 2). Result of the rabbit brain death model also show that efficient information for the quality evaluation of donor livers is lacking. Thus, it appears that in early brain death, the 'traditional criteria' may not be sufficiently efficient to identify an appropriate transplant donor. 
The proteomics analysis identified 52 different protein spots indicating that complex pathological changes occurred in the state of brain death. Ten significantly different proteins identified were classified into material metabolism and redox regulation, energy metabolism, cell proliferation and differentiation, lipid metabolism and detoxification and neurodevelopment. The appropriate systemic physiologic changes, which are considered to be principally a manifestation of brain death affecting all organs suitable for transplantation, were presumably the result of the ischemia/reperfusion injury $(8,10,11,26)$, followed by oxidative stress $(12,27)$, apoptosis $(28,29)$ and inflammatory response $(2,10,20)$. Evidence suggests that brain death results in the development of a systemic inflammatory response in the donor, which can damage all organs with deleterious impact on their function following transplantation.

RUNX1 is a nucleus gene whose major functions include cell proliferation and differentiation with the activation of PKC- $\theta$ and reactive oxygen species (30). Subsequent to the identification of the different RUNX1 protein by immunohistochemistry after proteomics and western blot analysis in the present study, we came to a primary conclusion that in addition to the development of brain death, a gradual decrease of RUNX1 expression was also induced. In other words, the expression of RUNX1 may be an indicator of the degree of brain death-induced liver injury, However, more investigations on the role of RUNX1 in brain death liver injury is required.

\section{Acknowledgements}

This investigation was supported by Research Fund for the Doctoral Program of Higher Education (20100141110016); Natural Science Fund of Hubei Province (2012FFA044; 2013CFB258); Science and technology projects of Wuhan city (2013060705010326; 2013060602010247).

\section{References}

1. Zhang SJ and Wang T: The influence of brain death on donor liver and the potential mechanisms of protective intervention. Front Med 5: 8-14, 2011.

2. Watts RP, Thom $\mathrm{O}$ and Fraser JF: Inflammatory signalling associated with brain dead organ donation: from brain injury to brain stem death and posttransplant ischaemia reperfusion injury. J Transplant 2013: 521369, 2013.

3. Machado C: The first organ transplant from a brain-dead donor. Neurology 64: 1938-1942, 2005.

4. Huang J, Mao Y and Millis JM: Government policy and organ transplantation in China. Lancet 372: 1937-1938, 2008.

5. Huang J, Mao Y, Wang Y, Zhang ZJ, Zhao MG and Liu Y: Modernization of the organ transplantation program in China. Transplantation 86: 1649-1652, 2008.

6. Huang J, Millis JM, Mao Y, Millis MA, Sang X and Zhong S: A pilot programme of organ donation after cardiac death in China. Lancet 379: 862-865, 2012.

7. Terasaki PI, Cecka JM, Gjertson DW and Takemoto S: High survival rates of kidney transplants from spousal and living unrelated donors. New Engl J Med 333: 333-336, 1995.

8. Avlonitis VS, Wigfield CH, Golledge HD, Kirby JA and Dark JH: Early hemodynamic injury during donor brain death determines the severity of primary graft dysfunction after lung transplantation. Am J Transplant 7: 83-90, 2007.
9. Stiegler P, Sereinigg M, Puntschart A, et al: Oxidative stress and apoptosis in a pig model of brain death (BD) and living donation (LD). J Transl Med 11: 244, 2013.

10. Avlonitis VS, Wigfield CH, Kirby JA and Dark JH: The hemodynamic mechanisms of lung injury and systemic inflammatory response following brain death in the transplant donor. Am J Transplant 5: 684-693, 2005.

11. Rostron AJ, Avlonitis VS, Cork DM, Grenade DS, Kirby JA and Dark JH: Hemodynamic resuscitation with arginine vasopressin reduces lung injury after brain death in the transplant donor. Transplantation 85: 597-606, 2008.

12. Leber B, Stadlbauer V, Stiegler P, et al: Effect of oxidative stress and endotoxin on human serum albumin in brain-dead organ donors. Transl Res 159: 487-496, 2012.

13. Vespa PM: Hormonal dysfunction in neurocritical patients. Curr Opin Crit Care 19: 107-112, 2013.

14. Ranasinghe AM and Bonser RS: Endocrine changes in brain death and transplantation. Best Pract Res Clin Endocrinol Metab 25: 799-812, 2011.

15. Hvas CL, Fenger-Eriksen C, Høyer S, Sørensen B and Tønnesen E: Hypercoagulation following brain death cannot be reversed by the neutralization of systemic tissue factor. Thromb Res 132: 300-306, 2013

16. Wu X, Du Z, Yu J, et al: Activity of factor VII in patients with isolated blunt traumatic brain injury: association with coagulopathy and progressive hemorrhagic injury. J Trauma Acute Care Surg 76: 114-120, 2014.

17. Kuecuek O, Mantouvalou L, Klemz R, et al: Significant reduction of proinflammatory cytokines by treatment of the brain-dead donor. Transplant Proc 37: 387-388, 2005.

18. Nijboer WN, Schuurs TA, van der Hoeven JA, et al: Effects of brain death on stress and inflammatory response in the human donor kidney. Transplant Proc 37: 367-369, 2005.

19. Koudstaal LG, 't Hart NA, Ottens PJ, et al: Brain death induces inflammation in the donor intestine. Transplantation 86: $148-154,2008$

20. Auråen H, Mollnes TE, Bjørtuft $\emptyset$, et al: Multiorgan procurement increases systemic inflammation in brain dead donors. Clin Transplant 27: 613-618, 2013.

21. Ignatova M, Guével B, Com E, et al: Two-dimensional fluorescence difference gel electrophoresis analysis of Listeria monocytogenes submitted to a redox shock. J Proteomics 79: 13-27, 2013.

22. Qiao B, Wang J, Xie J, et al: Detection and identification of peroxiredoxin 3 as a biomarker in hepatocellular carcinoma by a proteomic approach. Int J Mol Med 29: 832-840, 2012.

23. Pratschke J, Wilhelm MJ, Kusaka M, Laskowski I and Tilney NL: A model of gradual onset brain death for transplant-associated studies in rats. Transplantation 69: 427-430, 2000.

24. Van Der Hoeven JA, Moshage H, Schuurs T, Nijboer M, Van Schilfgaarde R and Ploeg RJ: Brain death induces apoptosis in donor liver of the rat. Transplantation 76: 1150-1154, 2003.

25. Sereinigg M, Stiegler P, Puntschart A, et al: Establishing a brain-death donor model in pigs. Transplant Proc 44: 2185-2189, 2012.

26. Weiss S, Kotsch K, Francuski M, et al: Brain death activates donor organs and is associated with a worse $\mathrm{I} / \mathrm{R}$ injury after liver transplantation. Am J Transplant 7: 1584-1593, 2007.

27. Golling M, Jahnke C, Fonouni H, et al: Distinct effects of surgical denervation on hepatic perfusion, bowel ischemia, and oxidative stress in brain dead and living donor porcine models. Liver Transpl. 13: 607-617, 2007.

28. Lau A, Arundine M, Sun HS, Jones M and Tymianski M: Inhibition of caspase-mediated apoptosis by peroxynitrite in traumatic brain injury. J Neurosci 26: 11540-11553, 2006.

29. Pérez López S, Vázquez Moreno N, Escudero Augusto D, et al: A molecular approach to apoptosis in the human heart during brain death. Transplantation 86: 977-982, 2008.

30. Giambra V, Jenkins CR, Wang H, et al: NOTCH1 promotes $\mathrm{T}$ cell leukemia-initiating activity by RUNX-mediated regulation of PKC- $\theta$ and reactive oxygen species. Nat Med 18: $1693-1698,2012$. 